\title{
Continuous Fraïssé Conjecture
}

\author{
Arnold Beckmann Martin Goldstern \\ Norbert Preining \\ Institute of Discrete Mathematics and Geometry \\ Vienna University of Technology, Austria \\ martin.goldstern@tuwien.ac .at \\ \{beckmann, preining\}@logic .at
}

Nov 5, 2004

\begin{abstract}
We will investigate the relation of countable closed linear orderings with respect to continuous monotone embeddability and will show that there are exactly $\aleph_{1}$ many equivalence classes with respect to this embeddability relation. This is an extension of Laver's result [Lav71, who considered (plain) embeddability, which yields coarser equivalence classes. Using this result we show that there are only $\aleph_{0}$ many different Gödel logics.
\end{abstract}

\section{Introduction}

The starting point of the present work was the question 'How many Gödel logics are there?' This question led us to the study of embeddability relations of (countable) linear orderings. The most important result in this field is Laver's classical result on the Fraïssé Conjecture [Lav71] which counts the number of scattered linear orderings with respect to bi-embeddability.

We will generalize Laver's method to deal not only with monotone but with continuous monotone embeddings, and come back to Gödel logics in Section 3 where we use this result to compute the number of Gödel logics. Gödel logics form a class of many-valued logics, which are one of the three fundamental $t$-norm based logics.

Our main result is that the set of countable closed linear orderings is betterquasi-ordered by strictly monotone continuous embeddability, even when we consider labeled countable closed linear orderings. As a corollary we derive that there are only countably many Gödel logics.

The main concepts in all these discussions are 'well-quasi orderings' and 'better-quasi-ordering', which have been introduced by Nash-Williams in a series of five papers in the 1960s NW63, NW64, NW65b, NW65a, NW68,

While considering embeddability relation of orderings, examples of infinite descending sequences, as well as infinite antichains can be given DM40, Sie50. In Fra48, Fraïsse made conjectures to the effect that the embeddability relation is more well behaved in the case of countable order types (later extended to 
scattered order types), stating that 'every descending sequence of countable order types is finite, and every antichain of countable order types is finite.' This conjecture was finally proved by Laver [Lav71].

\subsection{Basic concepts}

In our exposition we will mainly follow Rosenstein's textbook on linear orderings [Ros82], especially Chapter 10. To keep this article self-contained we will give all the necessary definition and cite some results, but ask the reader to consult the mentioned book for motivation, background and history of these concepts and results, as well as for the proofs.

Definition 1. (Ros82, 10.12-10.15) A quasi-ordering is a reflexive and transitive binary relation $\leq_{Q}$ on a set $Q$. With $<_{Q}$ we denote the strict part of $\leq_{Q}$, i.e. $p<_{Q} q$ iff $p \leq_{Q} q$ and $q \not_{Q} p$. We will often drop the index ${ }_{Q}$ if there is not danger of confusion.

We write $p \equiv_{Q} q$ iff both $p \leq_{Q} q$ and $q \leq_{Q} p$ hold. This is an equivalence relation; we write $Q / \equiv$ for the set of equivalence classes.

An infinite sequence $\vec{p}=\left\langle p_{n}: n<\omega\right\rangle$ is called good if there are indices $n<k$ with $p_{n} \leq p_{k} ; \vec{p}$ is called bad if it is not good. $\vec{p}$ is called an infinite descending chain if $p_{0}>_{Q} p_{1}>_{Q} p_{2}>_{Q} \ldots$ It is called an anti-chain of $Q$ if neither $p_{i} \leq_{Q} p_{j}$ nor $p_{j} \leq_{Q} p_{i}$ for $i \neq j$.

A set $Q$ is a well-quasi-ordering, denoted wqo, if any/all conditions in Lemma2 2 hold.

Lemma 2. ([Ros82], 10.16-10.17) Let $(Q, \leq)$ be partial order. Then the following are equivalent:

1. All sequences $\vec{q}=\left\langle q_{i}: i<\omega\right\rangle$ are good.

2. For all sequences $\vec{q}=\left\langle q_{n}: n<\omega\right\rangle$ there is an infinite subsequence $\left\langle q_{n}\right.$ : $n \in I\rangle$ which is either strictly increasing $\left(n<m\right.$ implies $\left.q_{n}<q_{m}\right)$ or constant $\left(n<m\right.$ implies $\left.q_{n} \equiv q_{m}\right)$.

3. There are no infinite antichains and no infinite decreasing chains in $Q$.

Definition 3. ( Ros82, 10.19) Given quasi-orderings $Q_{1}$ and $Q_{2}$, we define the quasi-ordering $Q_{1} \times Q_{2}$ by stipulating that $\left\langle p_{1}, p_{2}\right\rangle \leq\left\langle q_{1}, q_{2}\right\rangle$ if $p_{1} \leq_{Q_{1}} q_{1}$ and $p_{2} \leq_{Q_{2}} q_{2}$.

Lemma 4. (Ros82, 10.20) If $Q_{1}$ and $Q_{2}$ are wqo, then so is $Q_{1} \times Q_{2}$.

Definition 5. ([Ros82], 10.21, 10.24) Given a quasi-ordering $Q$, we define the quasi-ordering $Q^{<\omega}$, whose domain is the set of all finite sequences of elements of $Q$, by stipulating that $\left\langle p_{0}, p_{1}, \ldots, p_{n-1}\right\rangle \leq\left\langle q_{0}, q_{1}, \ldots, q_{m-1}\right\rangle$ if there is a strictly increasing $h: n \rightarrow m$ such that $a_{i} \leq_{Q} b_{h(i)}$ for all $i<n$.

We define the quasi-ordering $Q^{\omega}$ of $\omega$-sequences of elements of $Q$ by saying that $\left\langle p_{n}: n<\omega\right\rangle \leq\left\langle q_{n}: n<\omega\right\rangle$ if there is a strictly increasing $h: \omega \rightarrow \omega$ such that $a_{n} \leq_{Q} b_{h(n)}$ for all $n<\omega$.

Theorem 6. (Ros82, 10.23) If $Q$ is a wqo, then so is $Q^{<\omega}$. 
Definition 7. (Ros82, 10.31-10.33) If $c$ is a finite subset of $\mathbb{N}, d$ is any subset of $\mathbb{N}$, then we say that $d$ extends $c$ iff: $c=\{i \in d: i \leq \max c\}$, i.e., if $c$ is an initial segment (not necessarily proper) of $d$.

An infinite set $B$ of finite subsets of $\mathbb{N}$ is a block if every infinite subset $X$ of $\bigcup B:=\bigcup\{b: b \in B\}$ has an initial segment in $B$; that is, $X$ extends some element in $B$. A block $B$ is called a barrier if no two elements of $B$ are comparable w.r.t. inclusion.

A precedence relation $\triangleleft$ on a barrier $B$ is defined as follows: if $b_{1}$ and $b_{2}$ are elements of $B$, then we say that $b_{1}$ precedes $b_{2}$, written $b_{1} \triangleleft b_{2}$, if there are $i_{1}<i_{2}<\cdots<i_{m}$ such that $b_{1}=\left\{i_{1}, i_{2}, \ldots, i_{k}\right\}$ and $b_{2}=\left\{i_{2}, \ldots, i_{m}\right\}$ for some $k, 1 \leq k<m$. (In particular, $\{i\} \triangleleft\{j\}$ holds for all $i \neq j$.)

A function $f: B \rightarrow Q$ on a barrier $B$ is bad if, whenever $b_{1}, b_{2} \in B$ and $b_{1} \triangleleft b_{2}, f\left(b_{1}\right) \Varangle_{Q} f\left(b_{2}\right)$. Otherwise we say that $f$ is good.

Definition 8. (Ros82, 10.30) We say that $Q$ is a better-quasi-ordering, denoted bqo, if every $f: B \rightarrow Q$ is good, for every barrier $B$ of finite subsets of $\mathbb{N}$.

Remark. Every bqo is a wqo.

Proof. Use the barrier $B=\{\{n\}: n \in \mathbb{N}\}$.

Theorem 9. (Ros82, 10.38) If $Q$ is a bqo, then $Q^{<\omega}$ and $Q^{\omega}$ are bqo's.

Theorem 10. ( Ros82, 10.40) Let $B$ be a barrier and suppose that $B=B_{1} \cup B_{2}$ is a partition of $B$. Then there is a sub-barrier $C \subseteq B$ such that $C \subseteq B_{1}$ or $C \subseteq B_{2}$.

This ends the definitions and results we will need from Ros82.

Definition 11. A countable closed linear ordering, denoted cclo, is a countable closed subset of $\mathbb{R}$.

A strictly monotone continuous embedding $h$ (denoted smc-embedding) from a cclo $Q_{1}$ to a cclo $Q_{2}$ is an embedding $h: Q_{1} \rightarrow Q_{2}$ which is continuous on $Q_{1}$, i.e. whenever $\left(p_{n}\right)_{n \in \mathbb{N}}$ is a sequence in $Q_{1}$ converging to an element $p$ in $Q_{1}$, then $\left(h\left(p_{n}\right)\right)_{n \in \mathbb{N}}$ is a sequence in $Q_{2}$ converging to an element $h(p)$ in $Q_{2}$, and strictly monotone on $Q_{1}$, i.e. whenever $p, q \in Q_{1}$ with $p<_{Q_{1}} q$ then $h(p)<_{Q_{2}} h(q)$. (Here, "convergence" is always understood as convergence in the usual topology of $\mathbb{R}$.)

Definition 12 (labeled cclo). In addition to cclo, we will also have to consider the following notion: Fix a quasi-order $Q$ (usually a bqo, often a finite set or an ordinal). A $Q$-cclo is a function $A$ whose domain $\operatorname{dom} A$ is a cclo and whose range is contained in $Q$.

We write $A \preceq B$ ( $A$ is $Q$-smc-embeddable into $B$, or shortly $A$ is embeddable into $B$ ) iff there is a smc-embedding $h$ from $\operatorname{dom} A$ to $\operatorname{dom} B$ with the property $A(a) \leq_{Q} B(h(a))$ for all $a \in \operatorname{dom} A$.

If $Q$ is a singleton, then $A \preceq B$ reduces just to a smc-embedding from $\operatorname{dom} A$ to $\operatorname{dom} B$. If $Q=\{p, q\}$ is an antichain, or satisfies $p<q$, and $A(0)=A(1)=$ $q=B(0)=B(1), B(b)=p$ for all $b \neq 0,1$, then $A \preceq B$ means that there is a smc-embedding from $\operatorname{dom} A$ to $\operatorname{dom} B$ which moreover preserves 0 and 1 . Such embeddings will play an important rôle when we investigate Gödel sets and the number of Gödel logics. 


\section{$2 \quad Q$-labeled countable closed linear orderings}

Let us fix some bqo $(Q, \leq)$ for defining $Q$-cclo's.

Notation 13. We will use the following notation throughout the paper:

$$
L_{0}+L_{1}+L_{2} \ldots+p+\ldots+{ }_{2} L+{ }_{1} L+{ }_{0} L
$$

or

$$
\sum L_{i}+p+\sum{ }_{i}^{*} L
$$

When we write this term the following conditions are imposed:

- $p$ is an element of $Q$.

- All the $L_{i}$ and ${ }_{i} L$ are $Q$-cclo's.

- Either all $L_{i}$ are empty, or none of them are empty. Similarly, either all ${ }_{i} L$ are empty, or none of them are. We do not allow all $L_{i}$ and all ${ }_{i} L$ to be empty.

- $\operatorname{dom} L_{i}<\operatorname{dom} L_{i+1}<\operatorname{dom}_{i+1} L<\operatorname{dom}_{i} L$ for all $i$, where we write $A<B$ for " $A=\emptyset \vee B=\emptyset \vee \sup A<\inf B$ ". In particular, between the domains of any two of them (in the non-empty case) we can find an open interval.

- $\lim _{n \rightarrow \infty} a_{n}=\lim _{n \rightarrow \infty} a$, whenever $a_{n} \in \operatorname{dom} L_{n}$ and ${ }_{n} a \in \operatorname{dom}_{n} L$.

The meaning of such a term is the $Q$-cclo $L$ whose domain is the set $\bigcup_{i} L_{i} \cup$ $\{x\} \cup \bigcup_{i} L$ (where $x=\lim _{n \rightarrow \infty} a_{n}$ and/or $x=\lim _{n \rightarrow \infty}{ }_{n} a$ for any/all sequences satisfying $a_{n} \in \operatorname{dom} L_{n}$ and ${ }_{n} a \in \operatorname{dom}_{n} L$ ), and the function $L$ extends all functions $L_{i}$ and ${ }_{i} L$, and $L(x)=p$.

A "finite sum"

$$
L=L_{1}+\cdots+L_{n}
$$

is defined naturally: we allow this expression only when all $L_{i}$ are nonempty and satisfy $\max \operatorname{dom} L_{i}<\min \operatorname{dom} L_{i+1}$. In this case we let $\operatorname{dom}(L)=\bigcup_{i} \operatorname{dom}\left(L_{i}\right)$ and $L=\bigcup_{i} L_{i}$.

We will consider two slightly different operations ( $S, S^{\prime}$ below) to build complicated $Q$-cclo's from simpler ones. These two operations naturally correspond to two notions rk, $\mathrm{rk}^{\prime}$ of rank; a third rank that we occasionally use is the classical Cantor-Bendixson rank $\mathrm{rk}_{\mathrm{CB}}$ of a cclo.

Definition 14. Let $\mathscr{O}$ be a class of $Q$-cclo's. We let $S(\mathscr{O})$ ('sums from $\mathscr{O}$ ') be the set of all $Q$-cclo's which are finite sums of $Q$-cclo's from $\mathscr{O}$, plus the set of all $Q$-cclo's of the form

$$
L_{0}+L_{1}+L_{2} \ldots+p+\ldots+{ }_{2} L+{ }_{1} L+{ }_{0} L
$$

where $p \in Q$ and all $L_{n}$ and all ${ }_{n} L$ are in $\mathscr{O}$.

We let $S^{\prime}(\mathscr{O})$ ('unbounded sums from $\mathscr{O}^{\prime}$ ) be the set of all $Q$-cclo's of the form

$$
L_{0}+L_{1}+L_{2} \ldots+p+\ldots+{ }_{2} L+{ }_{1} L+{ }_{0} L
$$

where $p \in Q$ and all $L_{n}$ and all ${ }_{n} L$ are in $\mathscr{O}$, and

$$
\forall n \exists k>n L_{n} \preceq L_{k} \text { and } \forall n \exists k>n{ }_{n} L \preceq{ }_{k} L \text {. }
$$


As a consequence of the above definition we obtain for unbounded sums, that for all $n$ there are infinitely many $k>n$ such that $L_{n} \preceq L_{k}$ and ${ }_{n} L \preceq{ }_{k} L$.

\section{Definition 15.}

(a) Let $\mathscr{C}$ be the set of all $Q$-cclo's.

(b) Let $\mathscr{C}_{0}=\mathscr{C}_{0}^{\prime}$ be the set of all $Q$-cclo's with singleton or empty domain. For any $\alpha \leq \omega_{1}$ let

$$
\mathscr{C}_{\alpha+1}=S\left(\mathscr{C}_{\alpha}\right) \cup \mathscr{C}_{\alpha} \quad \mathscr{C}_{\alpha+1}^{\prime}=S^{\prime}\left(\mathscr{C}_{\alpha}^{\prime}\right) \cup \mathscr{C}_{\alpha}^{\prime}
$$

and for limit ordinals $\delta>0$ let $\mathscr{C}_{\delta}=\bigcup_{\alpha<\delta} \mathscr{C}_{\alpha}, \mathscr{C}_{\delta}^{\prime}=\bigcup_{\alpha<\delta} \mathscr{C}_{\alpha}^{\prime}$.

(c) For any $L \in \bigcup_{\alpha} \mathscr{C}_{\alpha}$ we define the rank of $L(\operatorname{rk}(L))$ as the first ordinal $\alpha$ at which $L$ occurs in $\mathscr{C}_{\alpha+1}$. Similar, we define $\operatorname{rk}^{\prime}(L)$ for $L \in \bigcup_{\alpha} \mathscr{C}_{\alpha}^{\prime}$ as the first ordinal $\alpha$ at which $L$ occurs in $\mathscr{C}_{\alpha+1}^{\prime}$.

(d) The set of all $Q$-cclo's whose domains are suborderings of $\operatorname{dom} L$ is denoted with $\mathscr{C}(L)$.

It is clear that $\mathscr{C}_{\omega_{1}}^{\prime} \subseteq \mathscr{C}_{\omega_{1}} \subseteq \mathscr{C}$. We will show that $\mathscr{C}=\mathscr{C}_{\omega_{1}}$, and that every order in $\mathscr{C}$ can be written as a finite sum of orders from $\mathscr{C}_{\omega_{1}}^{\prime}$.

Lemma 16. $\mathscr{C}=\mathscr{C}_{\omega_{1}}$. That is, for every $Q$-cclo $L$ there is a countable ordinal $\alpha$ such that $L \in \mathscr{C}_{\alpha}$.

Proof. We use the Cantor-Bendixson decomposition, more precisely we use induction on the Cantor-Bendixson rank of $V=\operatorname{dom} L$.

For every scattered closed set $V$ there is an ordinal $\operatorname{rk}_{\mathrm{CB}}(V)$ (the CantorBendixson rank of $V$ ) and a decomposition

$$
V=\bigcup_{\alpha \leq \mathrm{rk}_{\mathrm{CB}}(V)} \mathrm{CB}_{\alpha}(V)
$$

where $\mathrm{CB}_{0}(V)$ is the set of isolated points of $V$, and more generally each set $\mathrm{CB}_{\alpha}(V)$ is the set of isolated points of $V \backslash \bigcup_{\beta<\alpha} \mathrm{CB}_{\beta}(V)$, and $\mathrm{CB}_{\mathrm{rk}_{\mathrm{CB}}(V)}(V)$ is finite and nonempty.

Assume for the moment that $\mathrm{CB}_{\mathrm{rk}_{\mathrm{CB}}(V)}(V)$ is a singleton $\left\{x^{*}\right\}$. If $\operatorname{rk}_{\mathrm{CB}}(V)=$ 0 , then $L \in \mathscr{C}_{0}$. If $\operatorname{rk}_{\mathrm{CB}}(V)>0$, fix an increasing sequence $\left\langle x_{n}\right\rangle$ and a decreasing sequence $\left\langle_{n} x\right\rangle$, both with limit $x^{*}$, and $x_{n},{ }_{n} x \notin V$. Now it is easy to see that for all $\beta<\operatorname{rk}_{\mathrm{CB}}(V)$

$$
\mathrm{CB}_{\beta}\left(V \cap\left[x_{n}, x_{n+1}\right]\right)=\mathrm{CB}_{\beta}(V) \cap\left[x_{n}, x_{n+1}\right],
$$

so $\operatorname{rk}_{\mathrm{CB}}\left(V \cap\left[x_{n}, x_{n+1}\right]\right)<\operatorname{rk}_{\mathrm{CB}}(V)$, similarly for $V \cap\left[{ }_{n} x,{ }_{n+1} x\right]$. Now we can use the induction hypothesis.

If $\mathrm{CB}_{\mathrm{rk}_{\mathrm{CB}}(V)}(V)$ is not a singleton then we can write $V=V_{1}+\cdots+V_{n}$ for some finite $n$, with each $\mathrm{CB}_{\mathrm{rk}_{\mathrm{CB}}(V)}\left(V_{k}\right)$ a singleton, then proceed as above.

Definition 17. The set $\mathscr{C}^{\prime}:=\mathscr{C}_{\omega_{1}}^{\prime}$ is the smallest family of $Q$-cclo's which contains all the singletons and is closed under unbounded sums $S^{\prime}$.

Theorem 18. Let $L$ be a $Q$-cclo and assume that $(\mathscr{C}(L), \preceq)$ is a wqo. (See Definition 15(d).) Then $L$ is a finite sum of elements in $\mathscr{C}^{\prime}$. 
Proof. Induction on $\operatorname{rk}(L)$ : Assume that

$$
L=L_{0}+L_{1}+L_{2} \ldots+p+\ldots+{ }_{2} L+{ }_{1} L+{ }_{0} L
$$

where all the $L_{i}$ and ${ }_{i} L$ are in $\mathscr{C}^{\prime}$. Suppose that, for all but a finite number of $L_{i}$, each $L_{i}$ is embeddable in infinitely many $L_{j}$, and for all but a finite number of ${ }_{i} L$, each ${ }_{i} L$ is embeddable in infinitely many ${ }_{j} L$. Then $L$ can be written as

$L_{0}+\ldots L_{k-1}+\left(L_{k+0}+L_{k+1}+\cdots+p+\cdots+{ }_{l+1} L+{ }_{l+0} L\right)+{ }_{l-1} L+\cdots+{ }_{0} L$

where each summand is in $\mathscr{C}^{\prime}$.

Otherwise there are either infinitely many $L_{i}$ or ${ }_{i} L$ each embeddable in only finitely many $L_{j}$ or ${ }_{j} L$, resp. We then find a either a subsequence $\left\langle L_{h(n)}: n<\omega\right\rangle$ or $\left\langle_{h(n)} L: n<\omega\right\rangle$ no entry of which can be embedded in any subsequent entry. This bad sequence of suborderings of $L$ contradicts the hypothesis of the theorem.

Theorem 19. If $\left(\mathscr{C}^{\prime}, \preceq\right)$ is a bqo, then $(\mathscr{C}, \preceq)$ is a wqo.

Proof. We will show for all countable $L$ by induction on the $\operatorname{rank} \operatorname{rk}(L)$ (that is the rank w.r.t. the classes in $\mathscr{C}$ as defined in Definition 15 (c)), that the collection $\mathscr{C}(L)$ of $Q$-cclo's whose domains are suborderings of $\operatorname{dom} L$ is a wqo w.r.t smc-embeddability.

First we show that, if $K$ is in $\mathscr{C}(L)$, then $K$ can be written as $K=\sum J_{i}+$ $p+\sum_{i}^{*} J$, where all the $J_{i}$ and ${ }_{i} J$ are in $\mathscr{C}^{\prime}$. To prove this, observe that $L$ can be written as $\sum L_{i}+p+\sum_{i}^{*} L$ where the ranks of the $L_{i}$ and ${ }_{i} L$ are strictly less than the rank of $L$. Using the induction hypothesis, we see that $\left(\mathscr{C}\left(L_{i}\right), \preceq\right)$ and $\left(\mathscr{C}\left({ }_{i} L\right), \preceq\right)$ are wqo. If $\operatorname{dom} K$ is a sub ordering of $\operatorname{dom} L$, it can be written as $K=\sum K_{i}+q+\sum^{*}{ }_{i} K$ with $K_{i} \in \mathscr{C}\left(L_{i}\right)$ and ${ }_{i} K \in \mathscr{C}\left({ }_{i} L\right)$. Thus, by Theorem 18 . each $K_{i}$ and ${ }_{i} K$ can be written as finite sum of elements $J_{j}$ and ${ }_{j} J$ in $\mathscr{C}^{\prime}$, and $K$ as $J_{0}+J_{1}+J_{2} \ldots+q+\ldots+{ }_{2} J+{ }_{1} J+{ }_{0} J$.

Now consider a sequence $\left\langle K^{l}: l<\omega\right\rangle$, where each $K^{l}$ is a $Q$-cclo and subordering of $L$. We will repeatedly thin out this sequence, eventually arriving at a sequence which is good, which will show that our original sequence was good. After having thinned out the sequence $\left\langle K^{l}: l<\omega\right\rangle$ to a sequence $\left\langle K^{l_{i}}: i<\omega\right\rangle$, we will (for notational simplicity) relabel our index set so that we will also call the new sequence $\left\langle K^{l}: l<\omega\right\rangle$.

Each $K^{l}$ can be written as

$$
K^{l}=J_{0}^{l}+J_{1}^{l}+J_{2}^{l} \ldots+p^{l}+\ldots+{ }_{2} J^{l}+{ }_{1} J^{l}+{ }_{0} J^{l}
$$

where each of the summands is in $\mathscr{C}^{\prime}$. Using Lemma 2 we thin out our sequence to a new sequence (again called $\left\langle K^{l}: l<\omega\right\rangle$ ) such that $p^{j} \leq_{Q} p^{k}$ for all $j<k$.

By Theorem 9 we know that $\mathscr{C}^{\prime \omega}$ is a bqo, in particular a wqo. Consider the $\omega$-tuples $\mathcal{C}^{l}=\left\langle J_{0}^{l}, J_{1}^{l}, \ldots\right\rangle \in \mathscr{C}^{\prime \omega}$. Using Lemma 2 we can thin out our sequence to obtain a sequence satisfying $\mathcal{C}^{j} \preceq \mathcal{C}^{k}$ for any $j<k$.

We now apply the fact that $\mathscr{C}^{\prime \omega}$ is wqo to the sequence ${ }^{n} \mathcal{C}=\left\langle{ }_{0} J^{n},{ }_{1} J^{n}, \ldots\right\rangle \in$ $\mathscr{C}^{\prime \omega}$ to see that without loss of generality we may also assume ${ }^{j} \mathcal{C} \preceq{ }^{k} \mathcal{C}$ for all $j<k$.

Now pick any $n<m$, and consider the sums

$$
K^{n}=J_{0}^{n}+J_{1}^{n}+J_{2}^{n} \ldots+p^{n}+\ldots+{ }_{2} J^{n}+{ }_{1} J^{n}+{ }_{0} J^{n}
$$


and

$$
K^{m}=J_{0}^{m}+J_{1}^{m}+J_{2}^{m} \ldots+p^{m}+\ldots+{ }_{2} J^{m}+{ }_{1} J^{m}+{ }_{0} J^{m} .
$$

Write $x^{n}$ and $x^{m}$ for the central points of $K^{n}$ and $K^{m}$, respectively (i.e., $x^{n}=$ $\sup _{n} \bigcup_{i} \operatorname{dom} J_{i}^{n}=\inf _{n} \bigcup_{i} \operatorname{dom}_{i} J^{n}$, etc.)

We know $p^{n} \leq_{Q} p^{m}, \mathcal{C}^{n} \preceq \mathcal{C}^{m},{ }_{n} \mathcal{C} \preceq{ }_{m} \mathcal{C}$.

Thus, there are strictly increasing functions $g$ and $h$ from $\mathbb{N}$ to $\mathbb{N}$, such that for all $i, J_{i}^{n} \preceq J_{g(i)}^{m}$ and ${ }_{i} J^{n} \preceq{ }_{h(i)} J^{m}$. Let $\alpha_{i}$ and ${ }_{i} \alpha$ be functions that witness this, i.e., let $\alpha_{i}$ be a function mapping $\operatorname{dom} J_{i}^{n}$ to $\operatorname{dom} J_{g(i)}^{m}$ with $J_{i}^{n}(x) \leq$ $J_{g(i)}^{m}\left(\alpha_{i}(x)\right)$ for all $x \in \operatorname{dom} J_{i}^{n}$, and similarly ${ }_{i} J^{n}(x) \leq{ }_{g(i)} J^{m}\left({ }_{i} \alpha(x)\right)$ for all $x \in \operatorname{dom}_{i} J^{n}$,

Now define $\alpha: \operatorname{dom} K^{n} \rightarrow \operatorname{dom} K^{m}$ naturally: $\alpha$ extends all functions $\alpha_{i}$ and ${ }_{i} \alpha$, and $\alpha\left(x^{n}\right)=x^{m}$. Clearly $\alpha$ witnesses $K^{n} \preceq K^{m}$.

Finally, if $\left\{K_{i}: i<\omega\right\}$ is an arbitrary sequence, where each $K_{i}$ is in $\mathscr{C}$, then each $K_{i} \in \mathcal{C}(K)$ where $K=K_{0}+K_{1}+K_{2} \ldots+p+\ldots+{ }_{2} K+{ }_{1} K+{ }_{0} K$ for arbitrary $p$ and empty ${ }_{i} K$. According to the above remarks, the sequence $\left\{K_{i}: i<\omega\right\}$ must be good, so that $\mathcal{C}$ is a wqo.

Theorem 20. $\left(\mathscr{C}^{\prime}, \preceq\right)$ is a bqo.

We prove the Theorem by a series of lemmas. The first lemma holds for general quasi-orderings which are equipped with a rank function, it forms the main technical part of the proof of Theorem 20.

Let $(Q, \leq)$ be a quasi-ordering, and let $\rho$ be a rank function from $Q$ into the ordinals (i.e., a function satisfying $\rho(x) \leq \rho(y)$ whenever $x \leq y$ ). Let $\mathcal{F}$ denote the set of all functions $g: B \rightarrow Q$ where $B$ is a barrier of finite subsets of $\mathbb{N}$. (See Definition [7)

We say that $C$ is an extended sub-barrier of $B$ if $\cup C \subseteq \cup B$ and if every element of $C$ is an extension (not necessarily proper) of an element of $B$. $C$ is called a proper extended sub-barrier of $B$ if $C$ is an extended sub-barrier of $B$ and at least one element of $C$ properly extends some element of $B$. For two functions $g: B \rightarrow Q$ and $h: C \rightarrow Q$ in $\mathcal{F}$ we say that $h$ is shorter than $g$ if $C$ is a proper extended sub-barrier of $B$ and if $g$ and $h$ coincide on $B \cap C$, and if, whenever $c \in C$ properly extends $b \in B, h(c) \leq g(b)$ and $h(c)$ has lower rank than $g(b)$. The following Lemma can be extracted from the proof of Theorem 10.47 in Rosenstein [Ros82]. Recall from Definition 7 that a function $f: B \rightarrow Q$ is called bad if, whenever $b_{1}, b_{2} \in B$ and $b_{1} \triangleleft b_{2}, f\left(b_{1}\right) \not \leq f\left(b_{2}\right)$.

Lemma 21. If $\mathcal{F}$ contains some bad function, than it contains some minimal bad function, i.e. one which is minimal w.r.t. 'shorter'.

Proof. Assume for the sake of contradiction that $\mathcal{F}$ contains some bad function, but for any bad $g \in \mathcal{F}$ there is some bad $h \in \mathcal{F}$ which is shorter than $g$.

Let $g: B \rightarrow Q$ be bad. With $k(g)$ we denote the minimal $k$ such that there is a shorter $h: C \rightarrow Q$ and a $b \in B$ which is properly extended by some element in $C$ with $\max b \leq k$. Fix some witnesses $C, h$ and $b$ for $k(g)$. We define $D$ as the set of all $d \in B$ which do not have extensions in $C$ and which fulfill $d \subset[0, k(g)] \cup \cup C$. Obviously $C \cap D=\emptyset$.

First observe that for $d \in D$ we have $d \not \subset \cup C$ : Assume for the sake of contradiction that $d \subset \cup C$. Let $X$ be the infinite set $d \cup(\cup C \cap[\max d, \infty))$, then $X \subseteq \cup C$, hence there is some $c \in C$ which is extended by $X$. Since $X$ 
is also an extension of $d, c$ extends $d$ or vice versa. As $c$ extends some element in $B$ and $d \in B$, we have that $c$ cannot be properly extended by $d$ because $B$ is a barrier. But by definition of $D$ we also have that $c$ does not extend $d$. Contradiction.

Now, $B^{*}:=C \cup D$ is a barrier and $g^{*}: B^{*} \rightarrow Q$ defined by $g^{*}(c)=h(c)$ for $c \in C$ and $g^{*}(d)=g(d)$ for $d \in D$ is bad and shorter than $g$.

We verify these claims: First note that $\bigcup B^{*} \subseteq \bigcup C \cup[0, k(g)]$. For $B^{*}$ to be a block let $X \subseteq \cup B^{*}$ be infinite. There is some $d \in B$ which is extended by $X$ (as $B$ is a block, and $\bigcup B^{*} \subseteq \bigcup B$ ). If $d$ is not already in $B^{*}$ then, by definition of $D, d$ has some extension in $C$ which must be proper as $d \notin C$. Thus $d \subset \bigcup C$ and $\max d \geq k(g)$, hence $X \subseteq \bigcup C$ as $\bigcup B^{*} \subseteq \bigcup C \cup[0, k(g)]$. But then there is some $c \in C$ which is extended by $X$. Altogether this shows that $B^{*}$ is a block.

Assume that $B^{*}$ is not a barrier, then there must be $c \in C$ and $d \in D$ which are comparable. As $c$ is the extension of some element in $B$ and $d \in B$, we have $c \nsubseteq d$ because $B$ is a barrier. But $d \in D$ implies $d \not \subset \cup C$, hence $d \nsubseteq c$. Contradiction. Hence $B^{*}$ must be a barrier.

Obviously, $g^{*}$ is shorter than $g$, as $h$ already has been shorter than $g$. To verify that $g^{*}$ is bad we assume for the sake of contradiction that $c_{1} \triangleleft c_{2}$ and $g^{*}\left(c_{1}\right) \leq g^{*}\left(c_{2}\right)$. As $h$ is bad, $c_{1}$ and $c_{2}$ cannot be in $C$ at the same time. Similar with $g$, they cannot be in $D$ at the same time. If $c_{1} \in C$ and $c_{2} \in D$, then $c_{2} \not \subset \bigcup C$ which together with $c_{1} \triangleleft c_{2}, c_{1} \in C$ and the definition of $D$ shows $\max c_{1}<k(g)$, hence $c_{1} \in B$. Hence $g\left(c_{1}\right)=h\left(c_{1}\right)=g^{*}\left(c_{1}\right) \leq g^{*}\left(c_{2}\right)=g\left(c_{2}\right)$ contradicting that $g$ is bad. Therefore, $c_{1} \in D$ and $c_{2} \in C$. There is some $b_{2} \in B$ such that $b_{2}$ is extended by $c_{2}$. If $c_{1} \Varangle b_{2}$ then $b_{2} \subsetneq c_{1}$ which contradicts that $B$ is a barrier. Hence we have $c_{1} \triangleleft b_{2}$. But then $g\left(c_{1}\right)=g^{*}\left(c_{1}\right) \leq g^{*}\left(c_{2}\right)=$ $h\left(c_{2}\right) \leq g\left(b_{2}\right)$ contradicts that $g$ is bad. Altogether this shows that $g^{*}$ is bad.

We now define a sequence of bad elements $f_{n} \in \mathcal{F}$ in the following way. Let $f_{0}: B_{0} \rightarrow Q$ be some bad element in $\mathcal{F}$, and define recursively $B_{n+1}:=B_{n}^{*}$ and $f_{n+1}:=f_{n}^{*}$. Let $k_{n}:=k\left(f_{n}\right)$. Then $k_{n+1} \geq k_{n}$ because 'shorter' is transitive and $k_{n}$ is chosen minimal. Furthermore, $k_{n}=k_{m}$ for only finitely many $m$ since $\left\{b \in B_{n}: \max b=k_{n}\right\}$ is finite. Hence $\left\langle k_{n}: n<\omega\right\rangle$ is a non-decreasing unbounded sequence of natural numbers. Also observe that if $b \in B_{n}$ and $\max b<k_{n}$ and $n<m$ then $b \in B_{m}$, and if $b \in B_{m} \cap B_{n}$ then $f_{m}(b)=f_{n}(b)$.

Let $B:=\bigcup\left\{\cap\left\{B_{n}: n \geq m\right\}: m<\omega\right\}$. We show that $B$ is a barrier. Let $M:=\bigcap\left\{\bigcup B_{n}: n<\omega\right\} . M$ is infinite because $k_{n} \in M$ for all $n$. Let $X \subseteq M$ be infinite. Then for all $n<\omega$ we have $X \subseteq \cup B_{n}$, hence there is some $b_{n} \in B_{n}$ which is extended by $X$. If $b_{n+1}$ is a proper extension of $b_{n}$ then the rank of $f_{n+1}\left(b_{n+1}\right)$ is strictly smaller than the rank of $f_{n}\left(b_{n}\right)$, hence, for some $m$, $b_{n}=b_{m}$ for all $n \geq m$, i.e. $b_{m} \in \bigcap\left\{B_{n}: n \geq m\right\} \subseteq B$. In particular, $M \subseteq \bigcup B$ by taking $X:=M \cap[m, \infty)$ for $m \in M$. If $k \in \bigcup B$, then there is some $b \in B$ with $k \in b . \quad b \in B$ implies that there is some $m$ with $b \in \bigcap_{n>m} B_{n}$. Thus $k \in \bigcup B_{n}$ for all $n \geq m$. Also $k \in \bigcup B_{m} \subseteq \bigcup B_{m-1} \subseteq \cdots \subseteq \bigcup B_{0}$, hence $k \in M$. This shows $\bigcup B \subseteq M$. Thus $M=\bigcup B$ and $B$ is a block. Let $b, c \in B$, then $b, c \in B_{n}$ for some $n$, hence they are not comparable as $B_{n}$ is a barrier. Altogether this shows that $B$ is a barrier.

For $b \in B$ let $m_{b}:=\min \left\{m: b \in \bigcap\left\{B_{n}: n \geq m\right\}\right\}$. We define $f: B \rightarrow Q$ by $f(b):=f_{m_{b}}(b)$ and show that $f$ is minimal w.r.t. 'shorter' and bad. $f$ is shorter than $f_{n}$ for all $n$, because 'shorter' is transitive, $B$ is an extended subbarrier of $B_{n}$, if $b \in B \cap B_{n}$ then $m_{b} \leq n$ hence $f(b)=f_{m_{b}}(b)=f_{n}(b)$, and if $c \in B$ properly extends $b \in B_{n}$, then $m_{c}>n$ and $f(c)=f_{m_{c}}(c) \leq f_{n}(b)$ 
and $\rho(f(c))=\rho\left(f_{m_{c}}(c)\right)<\rho\left(f_{n}(b)\right) . f$ is bad, because if $b, c \in B$, and w.l.o.g. $m_{b} \leq m_{c}$, then $f(b)=f_{m_{c}}(b)$ and $f(c)=f_{m_{c}}(c)$ and $f_{m_{c}}$ is bad. By our general assumption there is some bad $f^{\prime}: B^{\prime} \rightarrow Q$ which is shorter than $f$. Then there are $b^{\prime} \in B^{\prime}$ and $b \in B$ such that $b^{\prime}$ properly extends $b$. Choose $n$ with $k_{n}>\max b^{\prime}$. Now $f^{\prime}$ is shorter than $f_{n}$ because $f$ is shorter than $f_{n}$ and 'shorter' is transitive. But this contradicts the minimality of $k\left(f_{n}\right)$. Hence our general assumption has been wrong, and the theorem is proved.

Recall that the $\operatorname{rank} \operatorname{rk}^{\prime}(L)$ of $L \in \mathscr{C}^{\prime}$ is given by the minimal $\alpha$ such that $L \in \mathscr{C}_{\alpha+1}^{\prime}$. A $\mathscr{C}^{\prime}$-term for $L \in \mathscr{C}^{\prime}$ with $\operatorname{rk}^{\prime}(L)>0$ is a faithful witness for $L \in \mathscr{C}^{\prime}$, i.e. a decomposition $L=\sum L_{i}+p+\sum{ }_{i}{ }_{i} L$ with all the $L_{i}$ and ${ }_{i} L$ in $\mathscr{C}^{\prime}$ and $\operatorname{rk}^{\prime}\left(L_{i}\right)<\operatorname{rk}^{\prime}(L)$ and $\operatorname{rk}^{\prime}\left({ }_{i} L\right)<\operatorname{rk}^{\prime}(L)$ for all $i$.

Lemma 22. Let $L$ and $K$ be in $\mathscr{C}^{\prime}$, with $L=\sum L_{i}+p+\sum{ }_{i}^{*} L$ and $K=$ $\sum K_{i}+q+\sum_{i}^{*} K$ being $\mathscr{C}^{\prime}$-terms of them. If $p \leq q$ and each $L_{i}$ is embeddable into some $K_{j}$ and each ${ }_{i} L$ is embeddable into some ${ }_{j} K$, then $L \preceq K$.

Proof. Let the assumptions of the lemma be fulfilled. Then there are $k_{i}, l_{i} \in \omega$ such that $k_{i}<k_{i+1}, l_{i}<l_{i+1}$ and $L_{i} \preceq K_{k_{i}}$ and ${ }_{i} L \preceq l_{i} K$ because $L, K$ are in $\mathscr{C}^{\prime}$. Fix smc-embeddings $\sigma_{i}: \operatorname{dom} L_{i} \rightarrow \operatorname{dom} K_{k_{i}}$ and ${ }_{i} \sigma: \operatorname{dom}_{i} L \rightarrow \operatorname{dom}_{l_{i}} K$ witnessing $L_{i} \preceq K_{k_{i}}$ resp. ${ }_{i} L \preceq{ }_{l_{i}} K$, and let $b:=\lim _{i}\left(\sup \operatorname{dom} L_{i}\right)$ and $c:=$ $\lim _{i}\left(\sup \operatorname{dom} K_{i}\right)$. We define a map $\sigma: \operatorname{dom} L \rightarrow \operatorname{dom} K$ by

$$
\sigma(a):= \begin{cases}\sigma_{i}(a) & \text { if } a \in \operatorname{dom}_{i} \\ i \sigma(a) & \text { if } a \in \operatorname{dom}_{i} L \\ c & \text { if } a=b\end{cases}
$$

Then $\sigma$ is a smc-embedding witnessing $L \preceq K$.

Proof of Theorem 20. Assume for the sake of contradiction that $\left(\mathscr{C}^{\prime}, \preceq\right)$ is not a bqo. By applying Lemma 21 we can find some $f: B \rightarrow \mathscr{C}^{\prime}$ which is bad and minimal w.r.t. 'shorter'. For each $b \in B$ we fix some $\mathscr{C}^{\prime}$-term $f(b)=$ $\sum L_{i}+p+\sum_{i}^{*} L$.

For any $a, b \in B$ with $a \triangleleft b$ we have that $f(a)=\sum L_{i}+p+\sum{ }_{i}{ }_{i} L \npreceq$ $\sum K_{i}+q+\sum{ }_{i}{ }_{i} K=f(b)$, hence, by applying Lemma 22, we see that at least one of the following holds:

(i) $p \not \leq q$

(ii) for some $i$ : $L_{i} \npreceq K_{j}$ for all $j$,

(iii) for some $i:{ }_{i} L \npreceq{ }_{j} K$ for all $j$.

By applying Theorem 10 we can find a sub-barrier $B^{\prime}$ such that one the cases (i), (ii), (iii) always happens on $B^{\prime}$. In the first case this would form a bad sequence in $(Q, \leq)$ which would contradict that $(Q, \leq)$ is a wqo. Thus, w.l.o.g. we may assume that for all $a, b \in B^{\prime}$ with $a \triangleleft b$ there is some $i$ such that $L_{i} \npreceq K_{j}$ for all $j$. Let $B^{\prime}(2):=\left\{b_{1} \cup b_{2}: b_{1}, b_{2} \in B^{\prime}\right.$ and $\left.b_{1} \triangleleft b_{2}\right\}$, then $B^{\prime}(2)$ is an extended sub-barrier of $B$. Define $g: B^{\prime}(2) \rightarrow \mathscr{C}^{\prime}$ by letting $g\left(b_{1} \cup b_{2}\right)$ be the first $L_{i}$ in $f\left(b_{1}\right)=\sum L_{i}+p+\sum{ }_{i}{ }_{i} L$ which is not embeddable into any $K_{j}$ from $f\left(b_{2}\right)=\sum K_{i}+q+\sum_{i}{ }_{i} K$. Then obviously $g$ is shorter than $f$. But also $g$ is bad, because if $b_{1} \cup b_{2} \triangleleft b_{3} \cup b_{4}$ then $b_{2}=b_{3}$ and hence $g\left(b_{1} \cup b_{2}\right) \npreceq g\left(b_{3} \cup b_{4}\right)$. This contradicts the minimality of $f$. 
Theorems 19 and 20 together yield the following result:

Corollary 23. $(\mathscr{C}, \preceq)$ is a wqo.

For the next corollary, we need the following two well-known properties of wqo's:

Lemma 24. Let $(Q, \leq)$ be a wqo with uncountable many $\equiv$-equivalence classes. Then there exists a 1-1 monotone map $f: \omega_{1} \rightarrow Q$.

Proof sketch. W.l.o.g. let each equivalence class of $Q / \equiv$ consist of one element. If each uncountable subset $Q^{\prime} \subseteq Q$ contains some element $q$ such that also $\left\{r \in Q^{\prime}: q \not \leq r\right\}$ is uncountable, then we can find sequence

$$
Q=Q_{0} \supseteq Q_{1} \supseteq Q_{2} \supseteq \cdots
$$

of uncountable sets with elements $q_{n} \in Q_{n}, Q_{n+1}:=\left\{r \in Q_{n}: q_{n} \not \leq r\right\}$. But then $q_{n} \not \leq q_{k}$ for all $n<k$, contradicting the assumption that $Q$ is wqo.

So there must be an uncountable subset $Q^{\prime} \subseteq Q$ such that for any $q \in Q^{\prime}$, the set $\left\{r \in Q^{\prime}: q \not \leq r\right\}$ is countable. But then we can easily find a copy of $\omega_{1}$ in $Q^{\prime}$.

Alternatively, start with any 1-1 sequence $\left\langle q_{i}: i \in \omega_{1}\right\rangle$ in $Q$; define a coloring $f:\left[\omega_{1}\right]^{2} \rightarrow 2$ by $f(i<j)=0$ iff $q_{i}<q_{j}$, and apply the Erdôs-Dushnik-Miller theorem $\omega_{1} \rightarrow\left(\omega_{1}, \omega\right)$. (See EHMR84, Theorem 11.1].)

Lemma 25. Let $Q$ be a countable bqo (or at least assume that $Q$ has only countably many $\equiv$-equivalence classes).

Then $Q^{\omega}$ (quasiordered as in Definition [5) has only countably many equivalence classes.

Proof. Part I: We first consider the set $Q^{*}$ of all sequences $\vec{q}=\left\langle q_{0}, q_{1}, \ldots\right\rangle \in Q^{\omega}$ satisfying

$$
\forall k \exists n>k: q_{k} \leq q_{n} .
$$

and show that this set is countable (modulo $\equiv$ ).

By Theorem $9 Q^{\omega}$ and hence also $Q^{*}$ is a wqo. Assume that $Q^{*}$ has uncountably many $\equiv$-classes, then by Lemma 24 we can find a sequence $\left\langle\vec{q}^{i}: i \in \omega_{1}\right\rangle$,

$$
\vec{q}^{i}=\left\langle q_{0}^{i}, q_{1}^{i}, \ldots\right\rangle \in Q^{*}
$$

with $i<j \Rightarrow \vec{q}^{i} \leq \vec{q}^{j}, \vec{q}^{j} \not \leq \vec{q}^{i}$.

Let $\alpha<\omega_{1}$ be so large such that every element of $Q$ which appears somewhere as $q_{n}^{j}$ is $\leq$ to some $q_{n^{\prime}}^{j^{\prime}}$ with $j^{\prime}<\alpha$.

We claim that $\vec{q}^{\alpha+1} \leq \vec{q}^{\alpha}$, which will be the desired contradiction.

By definition of $\alpha, \forall n \exists i<\alpha \exists n^{\prime}: q_{n}^{\alpha+1} \leq q_{n^{\prime}}^{i}$. So for every $n$ there is $n^{\prime \prime}$ with $q_{n}^{\alpha+1} \leq q_{n^{\prime \prime}}^{\alpha}$. Using $\vec{q}^{\alpha} \in Q^{*}$, we can find a sequence $k_{0}<k_{1}<\cdots$ with $q_{n}^{\alpha+1} \leq q_{k_{n}}^{\alpha}$ for all $n$, which means $\vec{q}^{\alpha+1} \leq \vec{q}^{\alpha}$.

Part II: For any sequence $\vec{q}=\left\langle q_{0}, q_{1}, \ldots\right\rangle \in Q^{\omega}$ we can find a natural number $N=N_{\vec{q}}$ such that $\forall k \geq N \exists n>k: q_{k} \leq q_{n}$, otherwise we get (as in the proof of Theorem [18) a contradiction to our assumption that $Q$ is a wqo. 
Now assume that $Q^{\omega} / \equiv$ is uncountable, then we can find a natural number $N^{*}$ and an uncountable family $\left\langle\vec{q}^{i}: i<\omega_{1}\right\rangle$ of pairwise nonequivalent sequences in $Q^{\omega}$ such that for all $i, N_{\vec{q}^{i}}=N^{*}$. Moreover, we may assume that all initial segments $\left\langle q_{0}^{i}, \ldots, q_{N^{*}}^{i}\right\rangle$ are equal to each other. Consider the tails $\left\langle q_{N^{*}+1}^{i}, q_{N^{*}+2}^{i}, \ldots\right\rangle \in Q^{\omega}$. By definition of $N_{\vec{q}^{i}}$, these tails are all in $Q^{*}$, defined in part I, above.

Hence we can find $i \neq j$ such that

$$
\left\langle q_{N^{*}+1}^{i}, q_{N^{*}+2}^{i}, \ldots\right\rangle \equiv\left\langle q_{N^{*}+1}^{j}, q_{N^{*}+2}^{j}, \ldots\right\rangle .
$$

But then also $\vec{q}^{i} \equiv \vec{q}^{j}$.

Corollary 26. Assume that our basic wqo $Q$ is countable. Then, for any set $\mathscr{O} \subseteq \mathscr{C}$ with $\mathscr{O} / \equiv$ countable we also have that $S^{\prime}(\mathscr{O}) / \equiv$ and even $S(\mathscr{O}) / \equiv$ are countable.

Proof. If $\left\langle L_{0}, L_{1}, \ldots\right\rangle \preceq\left\langle L_{0}^{\prime}, L_{1}^{\prime}, \ldots\right\rangle$ and $\left\langle{ }_{0} L,{ }_{1} L, \ldots\right\rangle \preceq\left\langle{ }_{0} L^{\prime},{ }_{1} L^{\prime}, \ldots\right\rangle$ and $p \leq$ $p^{\prime}$, then also

$$
L_{0}+L_{1}+\cdots+p+\cdots{ }_{1} L+{ }_{0} L \preceq L_{0}^{\prime}+L_{1}^{\prime}+\cdots+p+\cdots{ }_{1} L^{\prime}+{ }_{0} L^{\prime} .
$$

So the corollary follows from Lemma 25

Corollary 27. Assume that our basic wqo $Q$ is countable. W.r.t. continuous bi-embeddability there are exactly $\omega_{1}$ many equivalence classes of $Q$-cclo's.

Proof. It is easy to see (using the countable ordinals) that the number of equivalence classes is at least $\aleph_{1}$.

On the other hand, Corollary [26] implies that $\left|\mathscr{C}_{\alpha}\right| \leq \aleph_{0}$ for all $\alpha<\omega_{1}$, so $\left|\mathscr{C}_{\omega_{1}}\right| \leq \aleph_{1}$.

\section{Gödel logics}

Gödel logics are one of the oldest and most interesting families of many-valued logics. Propositional finite-valued Gödel logics were introduced by Gödel in Göd33 to show that intuitionistic logic does not have a characteristic finite matrix. They provide the first examples of intermediate logics (intermediate, that is, in strength between classical and intuitionistic logics). Dummett Dum59. was the first to study infinite valued Gödel logics, axiomatizing the set of tautologies over infinite truth-value sets by intuitionistic logic extended by the linearity axiom $(A \rightarrow B) \vee(B \rightarrow A)$. Hence, infinite-valued propositional Gödel logic is also called Gödel-Dummett logic or Dummett's LC. In terms of Kripke semantics, the characteristic linearity axiom picks out those accessibility relations which are linear orders.

Quantified propositional Gödel logics and first-order Gödel logics are natural extensions of the propositional logics introduced by Gödel and Dummett. For both propositional quantified and first-order Gödel logics it turns out to be inevitable to consider more complex truth value sets than the standard unit interval.

Gödel logics occur in a number of different areas of logic and computer science. For instance, Dunn and Meyer [DM71] pointed out their relation to 
relevance logics; Visser [Vis82] employed LC in investigations of the provability logic of Heyting arithmetic; three-valued Gödel logic has been used to model strong equivalence between logic programs. Furthermore, these logics have recently received increasing attention, both in terms of foundational investigations and in terms of applications, as they have been recognized as one of the most important formalizations of fuzzy logic Háj98.

Perhaps the most surprising fact is that whereas there is only one infinitevalued propositional Gödel logic, there are infinitely many different logics at the first-order level BLZ96, Baa96, Pre02. In the light of the general result of Scarpellini Sca62 on non-axiomatizability, it is interesting that some of the infinite-valued Gödel logics belong to the limited class of recursively enumerable linearly ordered first-order logics Hor69, TT84.

Recently a full characterization of axiomatizability of Gödel logics was given Pre03, where also the compactness of the entailment relation is discussed. But one of the most basic questions has been left open until now: How many Gödel logics are there? Lower bounds to this question have been given in Baa96. Pre02, and special subclasses of logics determined by ordinals have been discussed MTO90, but it was a long open question whether there are only countably many or uncountably many different Gödel logics.

\subsection{Syntax and Semantic}

In the following we fix a relational language $\mathscr{L}$ of predicate logic with finitely or countably many predicate symbols. In addition to the two quantifiers $\forall$ and $\exists$ we use the connectives $\vee, \wedge, \rightarrow$ and the constant $\perp$ (for 'false'); other connectives are introduced as abbreviations, in particular we let $\neg \varphi:=(\varphi \rightarrow \perp)$.

Originally, Gödel logics have been defined only based on the fixed truth value set $[0,1]$. But we can fix a (nearly) arbitrary subset of $[0,1]$ and consider the Gödel logic induced by this truth value set.

Definition 28 (Gödel set). A Gödel set is any closed set of real numbers, $V \subseteq[0,1]$ which contains 0 and 1 .

The (propositional) operations on Gödel sets which are used in defining the semantics of Gödel logics have the property that they are projecting, i.e. that the operation uses one of the arguments (or 1) as result:

Definition 29. For $a, b \in[0,1]$ let $a \wedge b:=\min (a, b), a \vee b:=\max (a, b)$,

$$
a \rightarrow b:= \begin{cases}1 & \text { if } a \leq b \\ b & \text { otherwise }\end{cases}
$$

The last operation is called 'Gödel's implication'. Note that

$$
(a \rightarrow b)=\sup \{x:(x \wedge a) \leq b\} ;
$$

in order theory this is expressed as 'the maps $x \mapsto(a \wedge x)$ and $y \mapsto(a \rightarrow y)$ are residuated'.

We define $\neg a:=(a \rightarrow 0)$, so $\neg 0=1$, and $\neg a=0$ for all $a>0$.

The semantics of Gödel logics, with respect to a fixed Gödel set as truth value set and a fixed relational language $\mathscr{L}$ of predicate logic, is defined using the 
extended language $\mathscr{L}^{M}$, where $M$ is a universe of objects. $\mathscr{L}^{M}$ is $\mathscr{L}$ extended with symbols for every element of $M$ as constants, so called $M$-symbols. These symbols are denoted with the same letters.

Definition 30 (Semantics of Gödel logic). Fix a Gödel set $V$ (and a relational language $\mathscr{L})$. A valuation $v$ into $V$ consists of

1. a nonempty set $M=M^{v}$, the 'universe' of $v$,

2. for each $k$-ary predicate symbol $P$, a function $P^{v}: M^{k} \rightarrow V$.

Given a valuation $v$, we can naturally define a value $v(A)$ for any closed formula $A$ of $\mathscr{L}^{M}$ For atomic formulas $\varphi=P\left(m_{1}, \ldots, m_{n}\right)$, we define $v(\varphi)=$ $P^{v}\left(m_{1}, \ldots, m_{n}\right)$, and for composite formulas $\varphi$ we define $v(\varphi)$ naturally by:

$$
\begin{aligned}
v(\perp) & =0 \\
v(\varphi \wedge \psi) & =\min (v(\varphi), v(\psi)) \\
v(\varphi \vee \psi) & =\max (v(\varphi), v(\psi)) \\
v(\varphi \rightarrow \psi) & =v(\varphi) \rightarrow v(\psi) \\
v(\forall x \varphi(x)) & =\inf \{v(\varphi(m)): m \in M\} \\
v(\exists x \varphi(x)) & =\sup \{v(\varphi(m)): m \in M\}
\end{aligned}
$$

(Here we use the fact that our Gödel sets $V$ are closed subsets of $[0,1]$, in order to be able to interpret $\forall$ and $\exists$ as inf and sup in V.)

For any closed formula $\varphi$ and any Gödel set $V$ we let

$$
\|\varphi\|_{V}:=\inf \{v(\varphi): v \text { a valuation into } V\}
$$

Remark. Note that the recursive computation of $v(\varphi)$ depends only on the values $M^{v}, P^{v}$ and not directly on the set $V$. Thus, if $V_{1} \subseteq V_{2}$ are both Gödel sets, and $v$ is a valuation into $V_{1}$, then $v$ can be seen also as a valuation into $V_{2}$, and the values $v(\varphi)$, computed recursively using (1)-(6), do not depend on whether we view $v$ as a $V_{1}$-valuation or a $V_{2}$-valuation.

If $V_{1} \subseteq V_{2}$, there are more valuations into $V_{2}$ than into $V_{1}$. Hence $\|\varphi\|_{V_{1}} \geq$ $\|\varphi\|_{V_{2}}$ for all closed $\varphi$.

Similarly, for any map $h: V_{1} \rightarrow V_{2}$, any valuation $v_{1}$ into $V_{1}$ induces a valuation $v_{2}$ into $V_{2}$ as follows:

$$
M^{v_{1}}=M^{v_{2}}, \quad P^{v_{1}}(\vec{m})=h\left(P^{v_{2}}(\vec{m})\right) .
$$

If $h: V_{1} \rightarrow V_{2}$ is a smc-embedding from $V_{1}$ into $V_{2}$ which moreover preserves 0 and 1 , and if $v_{2}$ is the valuation induced by $v_{1}$ and $h$, then it is easy to verify by induction on the complexity of the closed formula $\varphi$ that $v_{2}(\varphi)=h\left(v_{1}(\varphi)\right)$, and hence

$$
h\left(\|\varphi\|_{V_{1}}\right) \geq\|\varphi\|_{V_{2}}
$$

for all closed formulas $\varphi$.

Definition 31 (Gödel logics based on $V$ ). For a Gödel set $V$ we define the first order Gödel logic $\mathbf{G}_{V}$ as the set of all closed formulas of $\mathscr{L}$ such that $\|\varphi\|_{V}=1$. 
From the above remark it is obvious that if $h$ is as above or $V_{1} \subseteq V_{2}$, the Gödel logic $\mathbf{G}_{V_{2}}$ is a subset of $\mathbf{G}_{V_{1}}$.

Definition 32 (Submodel, elementary submodel). Let $v_{1}, v_{2}$ be valuations. We write $v_{1} \subseteq v_{2}\left(v_{2}\right.$ extends $\left.v_{1}\right)$ iff $M^{v_{1}} \subseteq M^{v_{2}}$, and for all $k$, all $k$-ary predicate symbols $P$ in $\mathscr{L}$, we have

$$
P^{v_{1}}=P^{v_{2}} \uparrow\left(M^{v_{1}}\right)^{k}
$$

or in other words, if $v_{1}$ and $v_{2}$ agree on closed atomic formulas.

We write $v_{1} \prec v_{2}$ if $v_{1} \subseteq v_{2}$ and $v_{1}(\varphi)=v_{2}(\varphi)$ for all $\mathscr{L}^{M^{v_{1}}}$-formulas $\varphi$.

Fact 33 (downward Löwenheim-Skolem). For any valuation $v$ (with $M^{v}$ infinite) there is a valuation $v^{\prime} \prec v$ with a countable universe $M^{v^{\prime}}$.

Definition 34. The only sub-formula of an atomic formula $P$ in $\mathscr{L}^{M}$ is $P$ itself. The sub-formulas of $\varphi \star \psi$ for $\star \in\{\rightarrow, \wedge, \vee\}$ are the subformulas of $\varphi$ and of $\psi$, together with $\varphi \star \psi$ itself. The sub-formulas of $\forall x \varphi(x)$ and $\exists x \varphi(x)$ with respect to a universe $M$ are all subformulas of all $\varphi(m)$ for $m \in M$, together with $\forall x \varphi(x)$ (or, $\exists x \varphi(x)$, respectively) itself.

The set of valuations of sub-formulas of $\varphi$ under a given valuation $v$ is denoted with

$$
\operatorname{Val}(v, \varphi)=\left\{v(\psi): \psi \text { sub-formula of } \varphi \text { w.r.t. } M^{v}\right\}
$$

Lemma 35. Let $v$ be a valuation with $v(\varphi)<b<1$ and $b$ does not occur in $\operatorname{Val}(v, \varphi)$. Let $v^{\prime}$ be the valuation with the same universe as $v$, defined by

$$
v^{\prime}(\psi)= \begin{cases}v(\psi) & \text { if } v(\psi)<b \\ 1 & \text { otherwise }\end{cases}
$$

for atomic subformulas $\psi$ of $\varphi$ w.r.t. $M^{v}$, and arbitrary for all other atomic formulas. Then $v^{\prime}$ is a valuation and $v^{\prime}(\varphi)=v(\varphi)$.

Proof. Let $h_{b}(a)=a$ if $a<b$ and $=1$ otherwise. By induction on the complexity of the formula $\psi$ we can easily show that $v^{\prime}(\psi)=h_{b}(v(\psi))$ for all subformulas $\psi$ of $\varphi$ w.r.t. $M^{v}$.

Lemma 36. Assume that $M \subset \mathbb{R}$ is a countable set and $P$ a perfect set. Then there is a smc-embedding from $M$ into $P$.

In Pre03 there is a proof of this lemma which was used to extend the proof of recursive axiomatizability of 'standard' Gödel logics (those with $V=[0,1]$ ) to Gödel logics with a truth value set containing a perfect set in the general case. Here we give a simple proof.

Proof. Since there are uncountable many disjoint sets of the form $\mathbb{Q}-x:=$ $\{q-x: q \in \mathbb{Q}\}$, there is some $x$ such that $M \cap(\mathbb{Q}-x)=\emptyset$, so also $(M+x) \cap \mathbb{Q}=\emptyset$. So we may assume that $M \cap \mathbb{Q}=\emptyset$. We may also assume $M \subseteq[0,1]$.

Since $P$ is perfect, we can find an smc-embedding $c$ from the Cantor set $C \subseteq[0,1]$ into $P$.

Let $i$ be the natural bijection from $2^{\omega}$ (the set of infinite $\{0,1\}$-sequences, ordered lexicographically) onto $C . i$ is an order preserving homeomorphism. 
For every $m \in M$ let $w(m) \in 2^{\omega}$ be the binary representation of $m$. Since $M$ does not contain any dyadic rational numbers, this representation is unique; moreover, the map $w$ is smc. Now $c \circ i \circ w$ is an smc embedding from $M$ into $P$.

$$
M \stackrel{w}{\longrightarrow} 2^{\omega} \stackrel{i}{\longrightarrow} C \stackrel{c}{\longrightarrow} P
$$

Lemma 37. Let $V$ be a truth value set with non-empty perfect kernel $P$, and let $W=V \cup[\inf P, 1]$, then the logics induced by $V$ and $W$ are the same, i.e. $\mathbf{G}_{V}=\mathbf{G}_{W}$.

Proof. As $V \subseteq W$ we have $\mathbf{G}_{W} \subseteq \mathbf{G}_{V}$. (Cf. Remark before Definition 31])

Now assume that $v_{W}(\varphi)<1$. Due to Fact 33, there is a $v_{W}^{\prime}$ such that $M^{v^{\prime}}$ is countable and $v_{W}^{\prime}(\varphi)=v_{W}(\varphi)$. The set $M:=\operatorname{Val}\left(v_{W}^{\prime}, \varphi\right)$ has cardinality at most $\aleph_{0}$, thus there exists a $b \in W$ such that $b \notin M, v_{W}^{\prime}(\varphi)<b<1$. According to Lemma 36] there is a smc-embedding $h$ from [inf $P, b] \cap(M \cup\{b\})$ into $P$. Define $v_{V}(\psi)$ for all atomic subformulas of $\varphi$ as follows:

$$
v_{V}(\psi)= \begin{cases}v_{W}^{\prime}(\psi) & \text { if } 0<v_{W}^{\prime}(\psi)<\inf P \\ h\left(v_{W}^{\prime}(\psi)\right) & \text { if } \inf P \leq v_{W}^{\prime}(\psi) \leq b \\ 1 & \text { otherwise }\end{cases}
$$

and 1 for all other atomic formulas. According to Lemma 35 we obtain that

$$
v_{V}(\varphi)= \begin{cases}v_{W}^{\prime}(\psi)<b<1 & \text { if } 0<v_{W}^{\prime}(\psi)<\inf P \\ h\left(v_{W}^{\prime}(\varphi)\right)<h(b) \leq 1 & \text { if } \inf P \leq v_{W}^{\prime}(\psi) \leq b\end{cases}
$$

thus $v_{V}(\varphi)<1$ and $\mathbf{G}_{V} \subseteq \mathbf{G}_{W}$.

Lemma 38. Let $V_{1}$ and $V_{2}$ be Gödel sets and $Q=\{0,1\}$ with $0<_{Q} 1$. Let $A_{1}$ and $A_{2}$ be $Q$-labeled cclos defined by $\operatorname{dom}\left(A_{i}\right)=V_{i}, A_{i}(0)=A_{i}(1)=1$ and $A_{i}(x)=0$ otherwise. If $A_{1}$ is (Q-smc-)embeddable into $A_{2}$, then the Gödel logic determined by $V_{1}$ is a superset of the Gödel logic determined by $V_{2}$.

Proof. In this case of a very simple labeling the property that $A_{1}$ is embeddable into $A_{2}$ reduces to the existence of a smc-embedding of $V_{1}$ into $V_{2}$ preserving 0 and 1. According to the Remark following Definition 30 this induces the reverse inclusion of the respective Gödel logics.

Corollary 39. The set of Gödel logics

(a) is countable

(b) is a (lightface) $\Sigma_{2}^{1}$ set

(c) is a subset of Gödel's constructible universe L.

Proof. (a) First note that the set of countable Gödel logics (i.e. those with countable truth value set), ordered by $\supseteq$, is a wqo. To see this, assume that $\left\langle\mathbf{G}_{n}: n \in \omega\right\rangle$ is a sequence of countable Gödel logics. Take the sequence of countable Gödel sets $\left\langle V_{n}: n \in \omega\right\rangle$ generating these logics and define the respective $Q$-labeled cclo (also denoted with $V_{n}$ ) with $Q=\{0,1\}, 0<_{Q} 1$ and 
$V_{n}(0)=V_{n}(1)=1$, and $V_{n}(x)=0$ otherwise. According to Corollary 23 this sequence of $Q$-labeled cclos must be good, hence there are numbers $n<m$ such that $V_{n}$ is smc-embeddable into $V_{m}$. Then Lemma 38 implies that $\mathbf{G}_{n}$ must be a superset of $\mathbf{G}_{m}$. This shows that the original sequence of Gödel logics $\left\langle\mathbf{G}_{n}: n \in \omega\right\rangle$ must be good, too.

As each countable Gödel logic is a subset of a fixed countable set (the set of all formulas), the family of countable Gödel logics cannot contain a copy of $\omega_{1}$. So by Lemma 24, the family of countable Gödel logics must be countable.

According to Lemma 37 any uncountable Gödel logic, i.e. Gödel logic determined by an uncountable Gödel set, such that 0 is not included in the prefect kernel $P$ of the Gödel set is completely determined by the countable part $V \cap[0, \inf P]$. So the total number of Gödel logics is at most two times the number of countable Gödel logics plus 1 for the logic based on the full interval, i.e. countable.

(b) First, note that the set

$$
\left\{(v, \varphi, v(\varphi)): M^{v}=\mathbb{N}\right\}
$$

is a Borel set, since we can show by induction on the quantifier complexity of $\varphi$ that the sets $\left\{(v, q): M^{v}=\mathbb{N}, v(\varphi) \geq q\right\}$ are Borel sets (even of finite rank).

Next, as set $G$ of formulas is a Gödel logic iff

There exists a closed set $V \subseteq[0,1]$ (say, coded as the complement of a sequence of finite intervals) such that:

- For every $\varphi \in G$, for every $v$ with $M^{v}=\mathbb{N}, v(\varphi)=1$, and

- For every $\varphi \notin G$, there exists $v$ with $M^{v}=\mathbb{N}, v(\varphi)<1$.

(We can restrict our attention to valuations $v$ with $v^{M}=\mathbb{N}$ because of Fact 33.)

Counting quantifiers we see that this is a $\Sigma_{2}^{1}$ property.

(c) follows from (a) and (b) by the Mansfield-Solovay theorem (see [Man70], [Mos80, 8G.1 and 8G.2]).

\section{Questions and future work}

Define $\omega_{1}^{G}$ as the smallest ordinal $\alpha$ such that: For every well-ordered Gödel set $V$ there is a well-ordered Gödel set $V^{\prime}$ of order type $<\alpha$ with $\mathbf{G}_{V}=\mathbf{G}_{V^{\prime}}$.

Define $\omega_{1}^{G C B}$ as the smallest ordinal $\alpha$ such that: For every Gödel set $V$ there is a Gödel set $V^{\prime}$ whose Cantor-Bendixson rank is $<\alpha$ with $\mathbf{G}_{V}=\mathbf{G}_{V^{\prime}}$.

By Corollary 39, both these ordinals are countable. Furthermore, $\omega_{1}^{G} \leq$ $\omega_{1}^{G C B}$. It would be interesting to describe the ordinals $\omega_{1}^{G}$ and $\omega_{1}^{G C B}$ by giving lower and upper estimates in terms of well-known closure ordinals, e.g. for inductive definitions and related reflection principles of set theory. Are they equal? Note that $\omega_{1}^{C K} \leq \omega_{1}^{G}$.

\section{References}

[Baa96] M. Baaz. Infinite-valued Gödel logics with 0-1-projections and relativizations. In P. Hájek, editor, Proc. Gödel'96, Logic Foundations of 
Mathematics, Computer Science and Physics - Kurt Gödel's Legacy, Lecture Notes in Logic 6, pages 23-33. Springer, 1996.

[BLZ96] M. Baaz, A. Leitsch, and R. Zach. Completeness of a first-order temporal logic with time-gaps. Theoret. Comput. Sci., 160(1-2):241270, June 1996.

[DM40] Ben Dushnik and E. W. Miller. Concerning similarity transformations of linearly ordered sets. Bull. Amer. Math. Soc., 46:322-326, 1940.

[DM71] J. M. Dunn and R. K. Meyer. Algebraic completeness results for Dummett's LC and its extensions. Z. Math. Logik Grundlagen Math, 17:225-230, 1971.

[Dum59] M. Dummett. A propositional logic with denumerable matrix. J. of Symbolic Logic, 24:96-107, 1959.

[EHMR84] Paul Erdôs, András Hajnal, Attila Máté, and Richard Rado. Combinatorial set theory: partition relations for cardinals, volume 106 of Studies in Logic and the Foundations of Mathematics. NorthHolland Publishing Co., Amsterdam, 1984.

[Fra48] Roland Fraïssé. Sur la comparaison des types d'ordres. C. R. Acad. Sci. Paris, 226:1330-1331, 1948.

[Göd33] K. Gödel. Zum Intuitionistischen Aussagenkalkül. Ergebnisse eines mathematischen Kolloquiums, 4:34-38, 1933.

[Háj98] P. Hájek. Metamathematics of Fuzzy Logic. Kluwer, 1998.

[Hor69] A. Horn. Logic with truth values in a linearly ordered Heyting algebra. Journal of Symbolic Logic, 34(3):395-409, 1969.

[Lav71] Richard Laver. On Fraïssé's order type conjecture. Ann. of Math. (2), 93:89-111, 1971.

[Man70] Richard Mansfield. Perfect subsets of definable sets of real numbers. Pacific J. Math., 35:451-457, 1970.

[Mos80] Yiannis N. Moschovakis. Descriptive set theory, volume 100 of Studies in Logic and the Foundations of Mathematics. North-Holland Publishing Co., Amsterdam, 1980.

[MTO90] P. Minari, M. Takano, and H. Ono. Intermediate predicate logics determined by ordinals. Journal of Symbolic Logic, 55(3):1099-1124, 1990.

[NW63] C. St. J. A. Nash-Williams. On well-quasi-ordering finite trees. Proc. Cambridge Philos. Soc., 59:833-835, 1963.

[NW64] C. St. J. A. Nash-Williams. On well-quasi-ordering lower sets of finite trees. Proc. Cambridge Philos. Soc., 60:369-384, 1964.

[NW65a] C. St. J. A. Nash-Williams. On well-quasi-ordering infinite trees. Proc. Cambridge Philos. Soc., 61:697-720, 1965. 
[NW65b] C. St. J. A. Nash-Williams. On well-quasi-ordering transfinite sequences. Proc. Cambridge Philos. Soc., 61:33-39, 1965.

[NW68] C. St. J. A. Nash-Williams. On better-quasi-ordering transfinite sequences. Proc. Cambridge Philos. Soc., 64:273-290, 1968.

[Pre02] N. Preining. Gödel logics and Cantor-Bendixon analysis. In M. Baaz and A. Voronkov, editors, Proceedings of LPAR'2002, LNAI 2514, pages 327-336, October 2002.

[Pre03] N. Preining. Complete Recursive Axiomatizability of Gödel Logics. PhD thesis, Vienna University of Technology, Austria, 2003.

[Ros82] Joseph G. Rosenstein. Linear orderings, volume 98 of Pure and Applied Mathematics. Academic Press Inc. [Harcourt Brace Jovanovich Publishers], New York, 1982.

[Sca62] B. Scarpellini. Die Nichtaxiomatisierbarkeit des unendlichwertigen Prädikatenkalkülus von Łukasiewicz. J. Symbolic Logic, 27:159-170, 1962.

[Sie50] Wacław Sierpiński. Sur les types d'ordre des ensembles linéaires. Fund. Math., 37:253-264, 1950.

[TT84] G. Takeuti and T. Titani. Intuitionistic fuzzy logic and intuitionistic fuzzy set theory. J. of Symbolic Logic, 49:851-866, 1984.

[Vis82] A. Visser. On the completeness principle: a study of provability in Heyting's Arithmetic. Annals of Math. Logic, 22:263-295, 1982. 